\title{
Intimate partner violence crisis in the COVID-19 pandemic: how can radiologists make a difference?
}

\author{
Simon Matoori ${ }^{1,2,3}$ (1) Bharti Khurana ${ }^{4} \cdot$ Marta Chadwick Balcom $^{5} \cdot$ Dow-Mu Koh $^{6}$ - Johannes M. Froehlich ${ }^{2,7}$. \\ Sonja Janssen ${ }^{8} \cdot$ Orpheus Kolokythas $^{9} \cdot$ Andreas Gutzeit $^{2,3,10}$
}

Received: 7 June 2020 / Accepted: 19 June 2020 / Published online: 30 June 2020

(C) European Society of Radiology 2020

\section{Key Points}

- The COVID-19 crisis resulted in a variety of physical and mental health issues beyond the viral infection itself, as indicated by an increase in domestic violence.

- Radiologists should be aware of typical intimate partner violence (IPV) injury patterns, actively ask potential IPV victims about the cause of injury, and be familiar with support systems for IPV victims of their institutions.

- Emergency and radiology departments should review their protocols for identifying and supporting IPV victims, and train their staff to work together to implement these measures during and beyond the COVID-19 crisis.

Our heads are round so our thoughts can change direction. (Francis Picabia)

The last few weeks have been challenging for radiologists. We had to prepare our departments for a new and highly contagious respiratory virus within a very short, and in some instances, no lag time before many seriously ill patients arrived $[1,2]$. In parallel, we had to rapidly learn from peer experience and scientific studies how to recognize the radiological patterns of this new disease and its complications. The primary focus of radiological departments was shifted to providing prompt and confident diagnosis of COVID-19 while adopting safety precautions and social distancing. However, this unprecedented

Simon Matoori

smatoori@seas.harvard.edu

Andreas Gutzeit

andreas.gutzeit@hirslanden.ch

1 John A. Paulson School of Engineering and Applied Sciences, Harvard University, Cambridge, MA, USA

2 Institute of Radiology and Nuclear Medicine and Breast Center St. Anna, Hirslanden Klinik St. Anna, Lucerne, Switzerland

3 Department of Radiology, Paracelsus Medical University, Salzburg, Austria

4 Department of Radiology, Brigham and Women's Hospital, Boston, MA, USA pandemic can also lead to other physical and mental health issues [3] which can present in the imaging department.

The COVID-19 pandemic has led to a combination of health and economic crisis, resulting from the combination of stock market crash in mid-February 2020, strong and sustained rise in unemployment, and the confinement of entire families to their domestic spaces following stay-at-home orders and school closures. The psychological stress affecting the domestically confined population led to outbreaks of violence, as observed by an increase in the numbers of domestic violence calls to the police departments in several cities in North America in the first months of the COVID-19 crisis (Table 1). This rise in intimate partner violence (IPV), defined by the World Health Organization as "any behavior within an

5 Community Health Intervention and Prevention Programs, Brigham and Women's Hospital, Boston, MA, USA

6 Department of Radiology, Royal Marsden Hospital, Downs Road, SM2 5PT Sutton, UK

7 Clinical Research Group, Klus Apotheke Zurich, Zurich, Switzerland

8 Clinic of Radiology and Nuclear Medicine, University Medical Center Mannheim, University of Heidelberg, Mannheim, Germany

9 Department of Radiology, University of Washington Medical Center, Seattle, WA, USA

10 Department of Chemistry and Applied Biosciences, Institute of Pharmaceutical Sciences, ETH Zurich, Zurich, Switzerland 
Table 1 North American cities whose police departments reported increased rates of domestic violence reports (all data directly communicated by the police departments)

\begin{tabular}{llll}
\hline City & Lockdown date & Investigated period & Reference period \\
\hline Boston (MA) & March 24, 2020 & March 24-April 20, 2020 & March 24-April 20, 2019 \\
Chicago (IL) & March 21, 2020 & March 16-March 29, 2020 & March 4-March 31, 2019 \\
Philadelphia (PN) & April 1, 2020 & March 1-March 31, 2020 & March 1-March 31, 2019 27\%* \\
Portland (OR) & March 23, 2020 & February 29-March 23, 2020 & March 12-March 23, 2019 +10\% \\
Seattle (WA) & March 23, 2020 & February 29-March 31, 2020 & February 28-March 31, 2019 \\
Toronto (ON) & March 17, 2020 & March 15-March 28, 2020 & March 1-March 28, 2019 + 20\%** \\
\hline
\end{tabular}

*Domestic violence-related aggravated assault

**Domestic violence-related arrests

intimate relationship that causes physical, psychological or sexual harm to those in the relationship," puts an issue into the spotlight which has existed in the fringe for many decades - visibly or invisibly. We see this increase in IPV as an opportunity for physicians in emergency and radiology departments to adapt and possibly expand measures to identify and support IPV victims during the COVID-19 crisis and beyond. Hence, this discourse aims to raise the awareness of the impact of the COVID-19 pandemic on IPV victims, and also to discuss how radiologists in Europe and around the world can make a difference for the victims.

Although several risk factors for IPV have been identified, such as female gender, low income, lower educational status, and unemployment of the partner, it is important to note that IPV may affect any person regardless of gender, religion, ethnicity, and socioeconomic status [4-7]. In addition, alcoholism and mental illness are also crucial health-related IPV risk factors $[6,7]$. In the general population, the lifetime prevalence of IPVrelated physical violence and/or unwanted sexual relations has been estimated at $23 \%$ among women [4]. IPV has a serious physical and emotional impact on the health of the victims [8].

Since the beginning of the COVID-19 crisis, many factors have negatively impacted on IPV-affected women seeking protection from their abuser. Emergency departments (ED) have been a safe haven for IPV victims, offering dedicated services for this patient population. However, because of perceived infection risk, IPV victims may prefer not to access the ED. Besides, as ED focused their services on COVID-19 patients, programs supporting IPV victims were often restricted or not available. Having anecdotal evidence that more severe IPV-related injuries were seen in ED during the stay-at-home measures, we assume that the threshold for IPV victims to seek assistance in the hospital was higher than usual.

Social distancing measures further affect the communication between IPV victims and their care givers. During telemedicine healthcare appointments, open communication is often not possible because of the difficulties in achieving physical separation from the abuser in the limited privacy of some homes. As a result, IPV screening questions in regular
non-IPV-related appointments may have to be omitted. Any counseling, which many IPV victims in an ongoing abusive relationship may receive in this scenario, also becomes less useful in this setting. Physical separation from the abuser is more difficult to achieve while stay-at-home measures are in place. During the initial phase of the lockdown in Boston, restraining orders were difficult to obtain due to the closure of courts and finding a space in a woman shelter became even more challenging. Many shelters, which had already been in high demand in pre-COVID-19 times, were not accepting new residents because of the need to apply de-densifying measures to maintain social distancing.

A recent large retrospective emergency visit screen for IPV revealed that the median age of victims was about 30 years, over $80 \%$ were women, and around half of the injuries occurred at home [9]. The most common diagnoses were contusion/abrasions, lacerations, strain/sprains, internal organ injuries, and fractures $[9,10]$. In general, most IPV fractures affect the face, neck, and head (nasal bones, orbit, maxillofacial bones, skull), with the middle third of the face affected in almost two-thirds of IPV cases [9-13]. However, the fractures most indicative for IPV are related to injuries of the upper and lower extremity, upper trunk, and head/neck [9]. The patient records may also give important clues as IPV victims generally visit the ED more often and undergo more imaging studies, and may contain terms like "contusion of soft tissue" and "superficial bruising" [10, 14, 15]. Many IPV injuries could easily be overlooked or misinterpreted as routine trauma in a busy emergency or radiology department. Therefore, we urge radiologists to be aware of the patterns of IPV-associated injuries and to carefully review the medical history even in common traumatic injuries. They should raise the alert in cases where there are inconsistencies between the injury and the reported history or where there are suspicious patient records. In practice, radiologists and emergency physicians may actively ask the potential IPV victim about the cause of injury and openly address the suspicion of IPV in interdisciplinary discussions to ensure that protective measures for the suspected IPV victim are introduced if needed. In the past, 
radiologists have been trained to be adept in recognizing another form of domestic violence: child abuse [16]. We believe current social tensions provoked by the COVID-19 crisis require radiologists to be attentive in identifying and supporting victims of abuse.

The global COVID-19 crisis has direct effects on families and communities, and we are witnessing how it can reshape and exacerbate existing social issues. However, this also presents an opportunity for radiologists to use their diagnostic competence to make a difference. You may ask yourself: is this our core task? We think so. In an age where there is growing influence from artificial intelligence, radiologists must move closer to the patients, make their approach more patient-oriented, and identify themselves as physicians who understand the complexities of patient care [17-25]. As patient-oriented physicians, radiologists should pro-actively engage with their patients in a way that allows them to identify and support IPV victims. Radiologists should appreciate the significant increase in IPV and the challenges that IPV victims face in these times of freedom of movement restrictions, economic hardship, and reduced hospital-based support systems. In our opinion, the COVID-19 crisis is the right moment for emergency and radiology departments to review their protocols for identifying and supporting IPV victims, and to train their staff to work together to implement these measures during and beyond the COVID-19 crisis.

Acknowledgments We thank the police departments of Boston (MA), Chicago (IL), Philadelphia (PN), Portland (OR), Seattle (WA), and Toronto (ON, Canada) for providing information on domestic violence. The police departments note that the results of ongoing investigations may change the classification of some of the domestic violence reports. The Boston police department further points to a change in their reporting system between 2019 and 2020 with potential implications on the comparability of the data. However, efforts were made to render the reported data as comparable as possible.

Funding information SM, MCB, DMK, JMF, SJ, OK, and AG state that they have not received any funding for this work. BK acknowledges funding provided by Stepping Strong Innovator Award and BCRISP, Brigham Health.

\section{Compliance with ethical standards}

Guarantor The scientific guarantor of this publication is Simon Matoori.

Conflict of interest The authors of this manuscript declare no relationships with any companies, whose products or services may be related to the subject matter of the article.

Statistics and biometry No complex statistical methods were necessary for this paper.

Informed consent Not applicable (editorial).
Ethical approval Not applicable (editorial).

Study subjects or cohorts overlap Not applicable.

Methodology

- Editorial

\section{References}

1. Gutzeit A, Li Q, Matoori S, Li B, Wang L (2020) What can European radiologists learn from the outbreak of COVID-19 in China? A discussion with a radiologist from Wuhan. Eur Radiol. https://doi.org/10.1007/s00330-020-06841-6

2. Devaraj A (2020) Important lessons for infection control in radiology departments during the COVID-19 pandemic. Eur Radiol. https://doi.org/10.1007/s00330-020-06873-y

3. Pfefferbaum B, North CS (2020) Mental health and the Covid-19 pandemic. N Engl J Med:NEJMp2008017. https://doi.org/10.1056/ NEJMp2008017

4. Breiding MJ, Black MC, Ryan GW (2008) Prevalence and risk factors of intimate partner violence in eighteen U.S. states/territories, 2005. Am J Prev Med 34:112-118. https://doi.org/10.1016/ j.amepre.2007.10.001

5. Thompson RS, Bonomi AE, Anderson M et al (2006) Intimate partner violence. prevalence, types, and chronicity in adult women. Am J Prev Med 30:447-457. https://doi.org/10.1016/j.amepre. 2006.01.016

6. Beydoun HA, Williams M, Beydoun MA, Eid SM, Zonderman AB (2017) Relationship of physical intimate partner violence with mental health diagnoses in the nationwide emergency department sample. J Women's Health (Larchmt) 26:141-151. https://doi.org/10. 1089/jwh.2016.5840

7. Cunradi CB, Todd M, Duke M, Ames G (2009) Problem drinking, unemployment, and intimate partner violence among a sample of construction industry workers and their partners. J Fam Violence 24:63-74. https://doi.org/10.1007/s10896-008-9209-0

8. Ellsberg M, Jansen HA, Heise L et al (2008) Intimate partner violence and women's physical and mental health in the WHO multicountry study on women's health and domestic violence: an observational study. Lancet 371:1165-1172. https://doi.org/10.1016/ S0140-6736(08)60522-X

9. Loder RT, Momper L (2020) Demographics and fracture patterns of patients presenting to US emergency departments for intimate partner violence. J Am Acad Orthop Surg Glob Res Rev 4: e2000009. https://doi.org/10.5435/jaaosglobal-d-20-00009

10. George E, Phillips CH, Shah N et al (2019) Radiologic findings in intimate partner violence. Radiology 291:62-69. https://doi.org/10. 1148/radiol.2019180801

11. Wu V, Huff H, Bhandari M (2010) Pattern of physical injury associated with intimate partner violence in women presenting to the emergency department: a systematic review and meta-analysis. Trauma Violence Abuse 11:71-82. https://doi.org/10.1177/ 1524838010367503

12. Hashemi HM, Beshkar M (2011) The prevalence of maxillofacial fractures due to domestic violence - a retrospective study in a hospital in Tehran, Iran. Dent Traumatol 27:385-388. https://doi.org/ 10.1111/j.1600-9657.2011.01016.x

13. Le BT, Dierks EJ, Ueeck BA, Homer LD, Potter BF (2001) Maxillofacial injuries associated with domestic violence. J Oral Maxillofac Surg 59:1277-1283. https://doi.org/10.1053/joms. 2001.27490

14. Kothari CL, Rhodes KV (2006) Missed opportunities: emergency department visits by police-identified victims of intimate partner 
violence. Ann Emerg Med 47:190-199. https://doi.org/10.1016/j. annemergmed.2005.10.016

15. Khurana B, Seltzer SE, Kohane IS, Boland GW (2020) Making the "invisible" visible: transforming the detection of intimate partner violence. BMJ Qual Saf 29:241-244

16. Nimkin K, Kleinman PK (1997) Imaging of child abuse. Pediatr Clin North Am 44:615-635. https://doi.org/10.1016/S00313955(05)70496-X

17. Recht M, Bryan RN (2017) Artificial intelligence: threat or boon to radiologists? J Am Coll Radiol 14:1476-1480. https://doi.org/10. 1016/j.jacr.2017.07.007

18. Gutzeit A, Fischmann A, Forstner R et al (2020) "I was seen by a radiologist, but unfortunately I can't remember the name and I still have questions. What should I do?" radiologists should give thoughts to improve service professionalism and patient esteem. Cancer Imaging 20:1-8. https://doi.org/10.1186/s40644-0200292-7

19. Gutzeit A, Heiland R, Sudarski S et al (2019) Direct communication between radiologists and patients following imaging examinations. Should radiologists rethink their patient care? Eur Radiol 29: 224-231. https://doi.org/10.1007/s00330-018-5503-2

20. Gutzeit A, Matoori S, Froehlich JM, et al (2016) Reduction in respiratory motion artefacts on gadoxetate-enhanced MRI after training technicians to apply a simple and more patient-adapted breathing command. Eur Radiol 26 (8):2714-2722
21. Matoori S, Froehlich JM, Breitenstein S et al (2019) Serum albumin, total bilirubin, and patient age are independent confounders of hepatobiliary-phase gadoxetate parenchymal liver enhancement. Eur Radiol 29(11):5813-5822

22. Matoori S, Froehlich JM, Breitenstein S et al (2016) Age dependence of spleen- and muscle-corrected hepatic signal enhancement on hepatobiliary phase gadoxetate MRI. Eur Radiol 26(6):18891894

23. Matoori S, Thian Y, Koh D-M et al (2017) Contrast-Enhanced CT Density Predicts Response to Sunitinib Therapy in Metastatic Renal Cell Carcinoma Patients. Transl Oncol 10 (4):679-685

24. Sudarski S, Haubenreisser H, Henzler T et al (2019) Incidence of transient interruption of contrast (TIC) - A retrospective singlecentre analysis in CT pulmonary angiography exams acquired during inspiratory breath-hold with the breathing command: "Please inspire gently!". PLOS ONE 14 (1):e0210473

25. Gutzeit A, Matoori S, Froehlich J, Koh D (2016) Reduction in Respiratory Motion Artifacts on Gadoxetate Acid-enhanced MR Images after Training Technicians. Radiology 279 (3):981-982

Publisher's note Springer Nature remains neutral with regard to jurisdictional claims in published maps and institutional affiliations. 